\title{
Research on Brain-Like Computer
}

\author{
Zhongzhi Shi \\ Key Laboratory of Intelligent Information Processing \\ Institute of Computing Technology, Chinese Academy of Sciences \\ Beijing 100190, China \\ shizz@ics.ict.ac.cn
}

After more than 60 years of development, the operation speed of computer is up to several hundred thousand billion $\left(10^{14}\right)$ times, but its intelligence level is extremely low. Studying machine which combines high performance and the people's high intelligence together becomes the effective way with high capacity and efficiency of exploring information processing. It will bring the important impetus to economic and social sustainable development, promotion of the information industry and so on to make breakthrough in the research of brain-like computer.

Mind is all mankind's spiritual activities, including emotion, will, perception, consciousness, representation, learning, memory, thinking, intuition, etc. Mind model is for explaining what individuals operate in the cognitive process for some thing in the real world. It is the internal sign or representation for external realistic world. If the neural network is a hardware of the brain system, then the mind model is the software of the brain system. The key idea in cognitive computing is to set up the mind model of the brain system, and then building brain-like computer in engineering through structure, dynamics, function and behavioral reverse engineering of the brain. This talk will introduce the research progress of brain-like computer, mainly containing intelligence science, mind modesl, neural columns, architecture of brain-like computers.

Intelligence Science is an interdisciplinary subject which dedicates to joint research on basic theory and technology of intelligence by brain science, cognitive science, artificial intelligence and others. Brain science explores the essence of brain, research on the principle and model of natural intelligence in molecular, cell and behavior level. Cognitive science studies human mental activity, such as perception, learning, memory, thinking, consciousness etc. In order to implement machine intelligence, artificial intelligence attempts simulation, extension and expansion of human intelligence using artificial methodology and technology. Research scientists coming from above three disciplines work together to explore new concept, new theory, new methodology. Intelligence science is a essential way to reach the human-level intelligence and point out the basic priciples for brain-like computer. 